
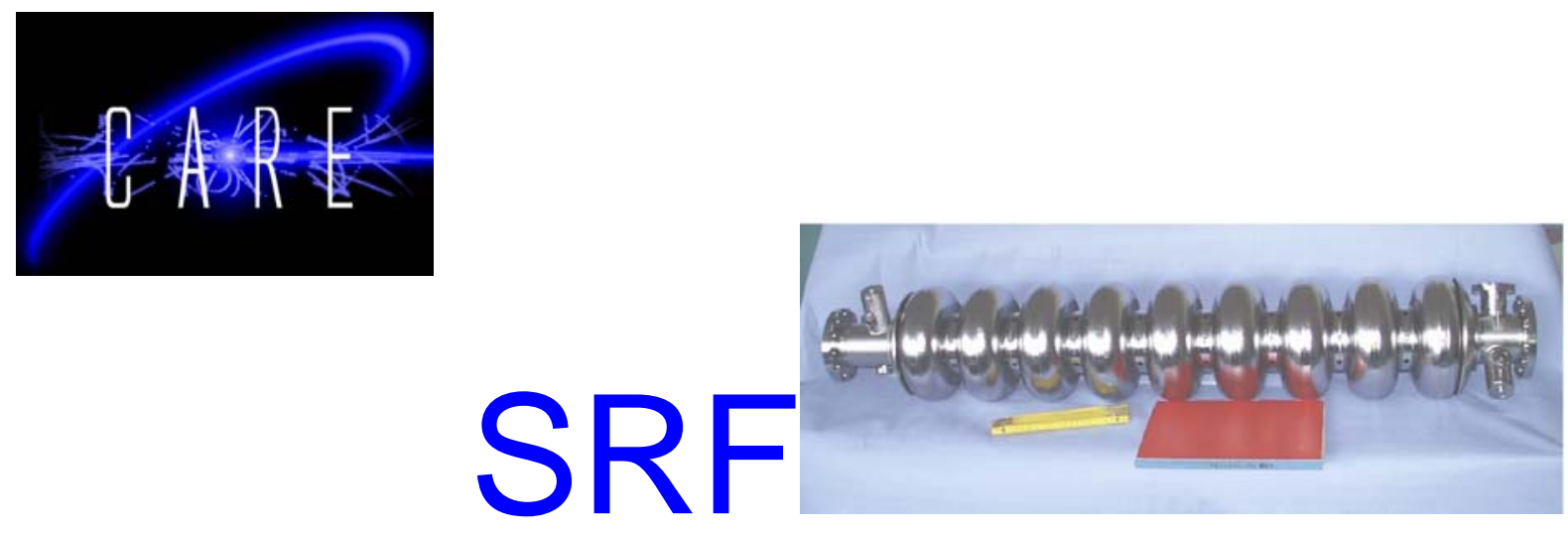

\author{
Super-conducting niobium films produced by means of UHV arc \\ J. Langner, M. J. Sadowski, K. Czaus, R. Mirowski, J. Witkowski \\ The Andrzej Soltan Institute for Nuclear Studies (IPJ), \\ L. Catani, A.Cianchi, R. Russo, S. Tazzari \\ University Tor Vergata and INFN-Roma2, \\ F.Tazzioli, Laboratori Nazionali dellOINFN \\ D. Proch, DESY \\ N.N. Koval, Y.H. Akhmadeev, HCEI, Tomsk, Russia
}

\begin{abstract}
The paper shows that high-quality super-conducting Nb films can be deposited by means of arc discharges under ultra-high vacuum conditions. The critical temperature $T c$ of Nb-films obtained with a planar cathodic arc Ultra High Vacuum (UHV) system, is very close to that of pure bulk niobium $T \mathrm{c}=9.26 \mathrm{~K}$ and the transition to super-conducting state is very narrow. The films have higher Residual Resistivity Ratio (RRR) values (up to 80) and larger grain sizes, as compared with sputtered $\mathrm{Nb}$-films deposited at the same temperature.
\end{abstract}

PACS: 52.77.Fv

Key words: vacuum arc, arc, superconductivity, thin films

Contribution to the 21st Symposium on Plasma Physics and Technology,

Prague, Czech Rep., June 2004

Work supported by the European Community-Research Infrastructure Activity under the FP6 "Structuring the European Research Area" programme (CARE, contract number RII3-CT2003-506395). 


\title{
Super-conducting niobium films produced by means of UHV arc
}

\author{
J. Langner, M. J. Sadowski, K. Czaus, R. Mirowski, J. Witkowski \\ The Andrzej Soltan Institute for Nuclear Studies (IPJ), \\ 05-400 Otwock-Swierk, Poland \\ e-mail: langner@ipj.gov.pl \\ L. Catani, A.Cianchi, R. Russo, S. Tazzari \\ University Tor Vergata and INFN-Roma2, \\ Via della Ricerca Scientifica 1, Roma, Italy \\ F.TAZZIOLI \\ Laboratori Nazionali dellOINFN, \\ Frascati, Italy \\ D. Proch \\ DESY, \\ 22603 Hamburg, Germany \\ N. N. Koval, Y. H. Akhmadeev \\ HCEI, \\ Tomsk, Russia
}

Received 7 May 2004

The paper shows that high-quality super-conducting $\mathrm{Nb}$ films can be deposited by means of arc discharges under ultra-high vacuum conditions. The critical temperature $T_{\mathrm{c}}$ of $\mathrm{Nb}$-films obtained with a planar cathodic arc Ultra High Vacuum (UHV) system, is very close to that of pure bulk niobium $T_{\mathrm{c}}=9.26 \mathrm{~K}$ and the transition to super-conducting state is very narrow. The films have higher Residual Resistivity Ratio (RRR) values (up to 80) and larger grain sizes, as compared with sputtered $\mathrm{Nb}$-films deposited at the same temperature.

PACS: 52.77.Fv

Key words: vacuum arc, arc, superconductivity, thin films

\section{Introduction}

In year 2000 the Ultra High Vacuum (UHV) arc technology was proposed as a possible alternative for depositing thin superconducting films of pure niobium on the inside surface of RF cavities for particle accelerators [1, 2]. Present, high field superconducting RF cavities are mainly made of high purity $(\mathrm{RRR} \geq 300)$ bulk $\mathrm{Nb}$, but were it possible to achieve the same accelerating fields of $\approx 35 \mathrm{MV} / \mathrm{m}$ with quality factors $(\mathrm{Q})$ in the $10^{10}$ range with superconducting film coated copper $(\mathrm{Nb} / \mathrm{Cu})$ cavities, the latter would prove a strong competitor. They would in fact offer several advantages such as better mechanical stability, insensitivity to external magnetic fields, possibly lower cost, better thermal stability, easier 
conditioning on the machine, easier connection to the cryostat. Cavities coated by cylindrical magnetron sputtering have been successfully demonstrated to be a valid alternative for relatively low accelerating fields: 352 four-cell cavities have been successfully operated during several years in LEP at up to $8 \mathrm{MV} / \mathrm{m}$, supplying a total of $3600 \mathrm{MV}$. Unfortunately, despite many years of R\&D at CERN [3], fast degradation of the Q with increasing field has so far prevented using them at higher fields. Since some experimental results seem to indicate, the $\mathrm{Q}$ degradation depends on the chemical and physical properties of the filmsubstrate system, the proposed UHV arc deposition is expected to improve the situation, as compared to magnetron sputtering. The UHV technique has advantages as follows: a high ionization ratio of the metallic plasma, and higher ion energy and a clean deposition process, due to absence of a working gas which makes for a purer film. The vacuum arc is in fact known to produce denser films, with much less defects such as voids and strongly bound to the substrate. Vacuum arc systems operating in initial vacuum of $10^{-4} \div 10^{-6} \mathrm{hPa}$ are commercially available for several coating applications. Being interested in depositing high purity materials, we have built at the Tor Vergata University of Rome and at IPJ Swierk the as far as we know first UHV (Ultra-High Vacuum) arc-based devices. They have planar and linear configurations operated at $\approx 10^{-10} \mathrm{hPa}$ base pressures. The systems have been used to study the deposition of superconducting $\mathrm{Nb}$ films. One device is equipped with a $90^{\circ}$ magnetic filter designed to eliminated micro-droplets of the material emitted from the cathode itself.

Recently obtained results are very promising but uniform coating of $1.5 \mathrm{GHz}$ copper cavities, e.g. of the TESLA type, using a filtered planar arc still needs further Research\&Development. An alternative being explored is that of using a linear geometry arc (linear arc) device that ideally meets the requirement of easily coupling to a single or multiple cell cavity. The cylindrical cathode of such a system can in fact be placed along the cavity axis like that of a cylindrical magnetron, with the arc discharge moving along it, either spontaneously or being magnetically driven. On the other hand, the problem of filtering out droplets becomes more complicate in this geometry. An unfiltered linear arc source prototype has been recently shown to work as expected and used to coat a cavity shaped chamber. In this paper we concentrate on the properties of superconducting thin films produced with a UHV planar arc apparatus, as well as on work in progress on the microdroplets problem. Since 2004 our experimental programme is financed in the framework of the European Union Coordinated Accelerator Research in Europe (CARE) Programme.

\section{UHV arc devices}

Cleanliness of the deposition process plays a crucial role in the formation of pure niobium films: for good superconducting film properties the partial pressures of water, nitrogen, oxygen, $\mathrm{CO}_{2}$, hydro carbides ect. must remain below $\approx 10^{-9} \mathrm{hPa}$ during the deposition. The pumping system must therefore be totally oil-free and all parts of the deposition system must be designed and built in accordance with UHV technology requirements. In our case, all vacuum chamber components and accessories, as well as all vacuum connections, were fabricated using only high purity materials: stainless steel, oxygen free (OFHC) copper and high quality ceramics shielded from the arc. The cathode and all parts acces- 
sible to the arc are made of $250 \mathrm{RRR} \mathrm{Nb}$ only. A base pressure of the order of $10^{-10} \mathrm{hPa}$ is reached after one night baking of the whole system at $150^{\circ} \mathrm{C}$. To check the composition of the residual gases before and during coating, the vacuum chambers are equipped with Residual Gas Analyzers (RGA). Details of the two UHV planar arc systems in operation at the Tor Vergata University, shown in Fig. 1, have been described elsewhere [4]. One of them is equipped with a $90^{\circ}$ magnetic filter, so that films produced with and without filtering can be compared. A schematic drawing of the UHV linear arc system recently put in operation at IPJ Swierk is shown in Fig. 2.

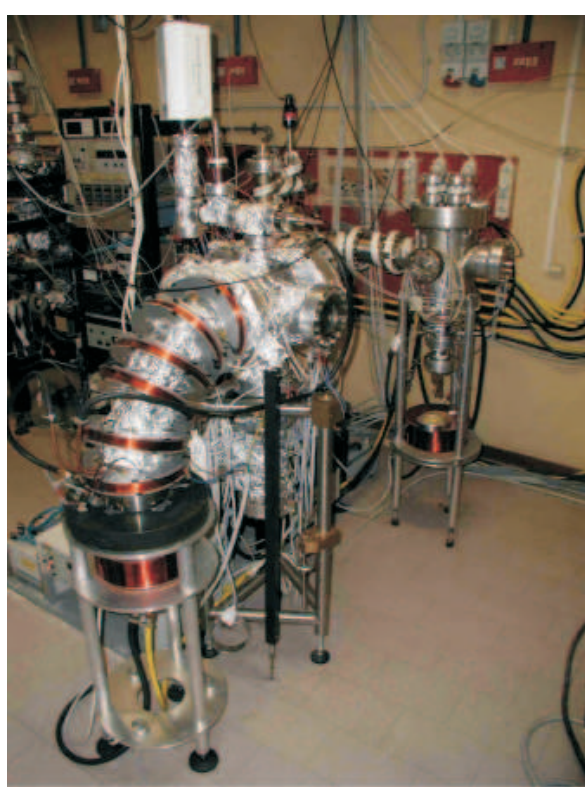

Fig. 1. Photograph of two UHV planar arc sources in Tor Vergata University.

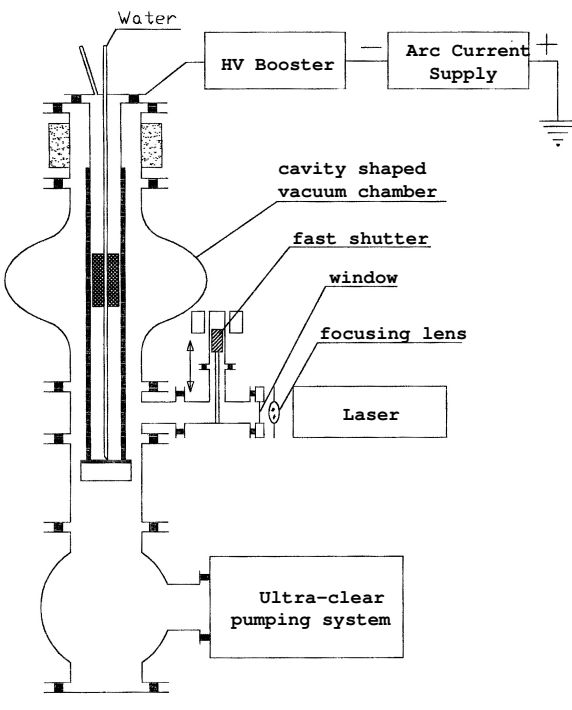

Fig. 2. Schematic drawing of new UHV setup with linear arc source designed and put recently into operation in IPJ Swierk.

Vacuum arc deposition is in principle a very pure process since the arc discharge burns in an atmosphere of evaporated cathode material only. In practice though a number of auxiliary systems must be carefully designed in order not to contaminate the vacuum enclosure, the main one being of the arc triggering system. It is well known that, to start the arc, a pre-plasma burst must be produced forming a high-conductivity path between cathode and anode. This can be done in many different ways [5], all of which must deliver several $\mathrm{mJ}$ of energy. Having tested several triggering techniques from the point of view of operational reliability and cleanliness, we found that only a few are compatible with UHV, the best being laser ignition: a laser focused on the cathode through a window ignites the arc without introducing any additional impurity. Two different lasers have been successfully tested: on the planar arc in Rome, a $60 \mathrm{~mJ}$ Nd:YAG laser with a $5 \mathrm{~ns}$ pulse width and up to $20 \mathrm{~Hz}$ repetition rate and, on the linear arc in Swierk, a (re-adapted) $700 \mathrm{~mJ}$ ruby laser, with $50 \mathrm{~ns}$ pulse width and relatively low repetition rate. As discussed below, laser 
ignition was decisive in improving the film superconducting properties.

\section{Formation of niobium superconducting films}

In order to coat sapphire and $\mathrm{Cu}$ substrates in the planar geometry, the samples were mounted on a special holder consisting of a massive $\mathrm{Cu}$ (OFHC) flange placed at a distance of about $50 \mathrm{~cm}$ from the cathode. It could keep up to 6 samples and it was kept at a constant temperature during the whole deposition process. A rotating shutter mounted in front of the sample holder allowed varying the coating conditions of different samples without breaking the vacuum. The sample holder was electrically insulated from the vacuum chamber and a $20 \div 100 \mathrm{~V}$ bias potential could be applied to the substrates. The lowest possible arc current for stable operation in the present DC mode was found to be about $60 \mathrm{~A}$ while the available cooling power did set a $140 \mathrm{~A}$ upper limit to the arc current. The deposition rate achievable with the unfiltered planar arc source can be very high, its precise value depending on many factors such as arc current, cathode material, geometry, applied fields, ect. In our planar system the deposition rate was $\approx 10 \mathrm{~nm} / \mathrm{s}$ while, with the present, non optimized configuration of our filtered apparatus it dropped by a factor of $\approx 5$. The sample temperature during depositions was recorded by means of a set of thermocouples. Most of the samples were deposited at close to room temperature and only a few at higher $\left(100 \div 200^{\circ} \mathrm{C}\right)$ temperature. As above mentioned, the base pressure in our systems was in the $10^{-10} \mathrm{hPa}$ range, increasing up to $10^{-6} \div 10^{-7} \mathrm{hPa}$ as soon as the arc discharge started, and remaing almost stable at the latter value throughout the deposition. The residual gas pressure during the discharge was found to be almost exclusively due to hydrogen, its partial pressure being usually more than 3 orders of magnitude higher than that of other contaminants. The presence of almost pure hydrogen is attributed to the bulk $\mathrm{Nb}$ cathode being a practically infinite source of this gas. Because all other gases only come from the chamber walls surface their partial pressures drops below the detection limit of our instrument after a few minutes of operation because of the getter action of the $\mathrm{Nb}$ film that forms on them. Only at most the first few nm of the film next to the substrate, can therefore become, albeit slightly, contaminated.

\section{Properties of the deposited film}

The critical temperature, $T_{\mathrm{c}}$, of the deposited material is very sensitive to impurities to the point that very small amounts of oxygen in the $\mathrm{Nb}$ film can lower its $T_{\mathrm{c}}$ value significantly. The Residual Resistivity Ratio (RRR - defined as the Resistivity at room temperature divided by the Resistivity measured at $10 \mathrm{~K}$ ) for $\mathrm{Nb}$ is also very sensitive to impurities. Typical RRR values for $\mathrm{Nb}$ films deposited by sputtering at a room temperature range from 2 to 10 , and films with $\mathrm{RRR} \approx 25$ are obtained either using $\mathrm{Kr}$ instead of $\mathrm{Ar}$ as the auxiliary gas, or raising the substrate temperature to $\approx 250^{\circ} \mathrm{C}$. UHV $\operatorname{arc}$ deposited samples, mainly upon the sapphire substrates, have therefore been characterized by measuring their RRR values and critical temperature $T_{\mathrm{c}}$. Their morphology was also studied using X-ray diffraction and the Atomic Force Microscope (AFM). The RRR of our $1.5 \mu \mathrm{m}$ thick $\mathrm{Nb}$ films deposited on room temperature sapphire substrates, under the 
typical UHV conditions described above, ranges from 20 up to 50, a typical value being 40 . A record value of $R R R=80$ was obtained by heating the substrate to $150^{\circ} \mathrm{C}$. The transition to superconducting state for this sample is shown in Fig. 3.

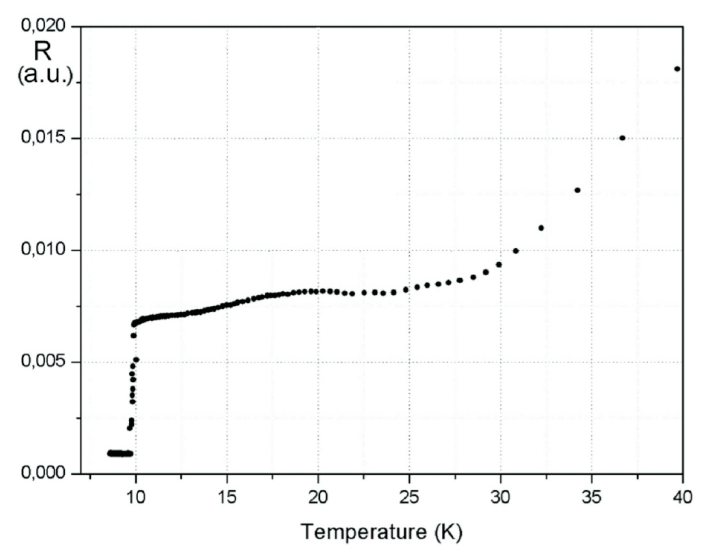

Fig. 3. The transition to superconducting state for the thin Niobium film deposited on sapphire.

Critical temperature $\left(T_{\mathrm{c}}\right)$ and critical current density $\left(J_{\mathrm{c}}\right)$ of the films were measured using an inductive method. Typical results are shown in Fig. 4 for several samples.

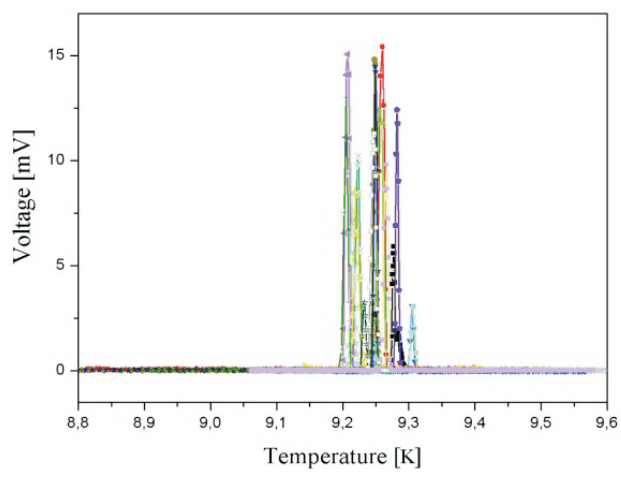

Fig. 4. Critical temperature measurements on $\mathrm{Nb}$ samples deposited by UHV arc.

Differences in $T_{\mathrm{c}}$ compared to the high purity bulk $\mathrm{Nb}$ value of $9.26 \mathrm{~K}$ are small and can be in part attributed to small temperature differences between thermometer and sample. No dependence is observed on either film thickness, in the $0.1 \div 2 \mu \mathrm{m}$ range, or on arc current. This indicates that our films are less stressed than magnetron sputtered ones, an indication confirmed by the X-ray Diffractometry (XRD) analysis (in a $\Theta / 2 \Theta$ configuration) yielding a lattice parameter in the $0.3308 \div 0.3318 \mathrm{~nm}$ range, a value close to the $0.3306 \mathrm{~nm}$ of bulk $\mathrm{Nb}$. A further confirmation comes from the sharp transition widths $(<0.02 \mathrm{~K})$, close to the $\approx 0.01 \mathrm{~K}$ value of pure bulk $\mathrm{Nb}$. 


\section{Microdroplets}

As earlier mentioned, the main inconvenience of arc coating is the production of microdroplets that, unless filtered out of the plasma, are embedded in the growing film and deposited on its surface. $\mathrm{n}$ our case, microdroplets being of high purity molten $\mathrm{Nb}$ are not expected to contaminate the film but do increase its surface roughness and, in a high electric field environment, may become fieldemitters. The presence of microdroplets on the surface of our films was studied by optical and electron microscopy and by roughness measurements. Using a 500× magnification optical microscope pictures of the sample surface were taken at 10 different randomly chosen locations and analyzed using a LabView computer code that measured and recorded the number and size of the droplets present in the microscope (fixed area) field of view. Fig. 5 shows the distribution of the droplet density as a function of the droplet size on the surface of 4 different samples deposited under comparable conditions.

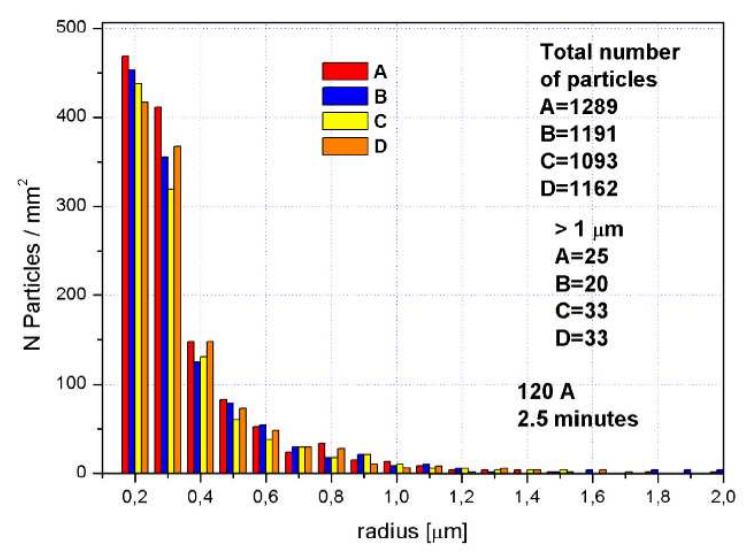

Fig. 5. Typical distribution of the droplet density on the surface of samples A, B, C and D, all deposited under same conditions. The lower symbol $>1 \mu \mathrm{m}$ means that we counted microdroples of diameter larger than $>1 \mu \mathrm{m}$

Note that the dimensions most observed droplets are comparable to size $(200 \div 300 \mathrm{~nm})$ of the $\mathrm{Nb}$ film grains whose boundaries can be seen in the picture of Fig. 6. One thus expects that many such droplets have become embedded in the growing film. Numerous larger droplets $(>1 \mu \mathrm{m})$ are also present that may become sources of field emission from the coated surface of an RF cavity. A few of such large droplets is shown in Fig. 6.

Magnetic filtering drastically reduces the number and the dimension of microparticles. Quantitative data on the reduction factor have not so far been obtained because, after filtering, the number of droplets in the observed field is often below the detection threshold, as shown in Fig. $7 \mathrm{~b}$. 


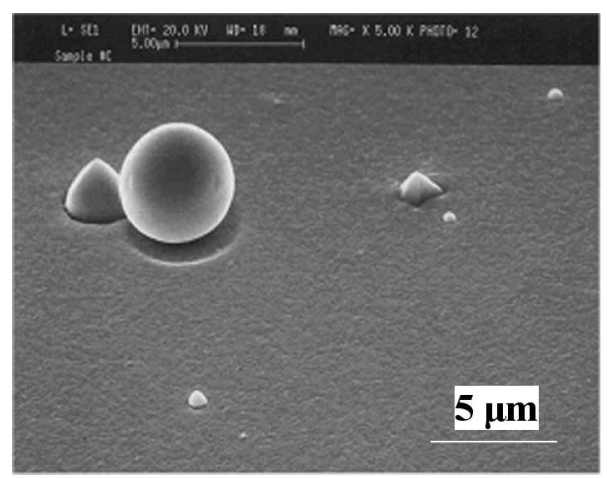

Fig. 6. SEM picture in tilted view for a non-filtered niobium film deposited on sapphire.

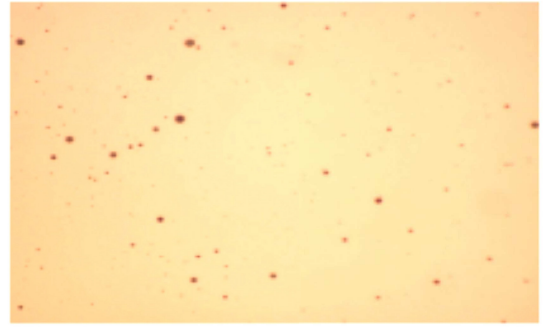

a)

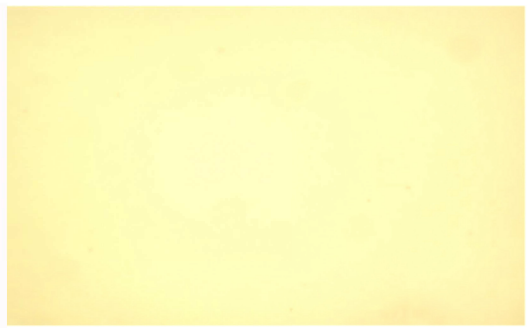

b)

Fig. 7. Microphotographs of samples deposited with unfiltered (a) and filtered (b) UHV arc systems, with same arc parameters $(500 \times$ magnification $)$

\section{Summary}

Several cathodic arc sources working in UHV conditions have been designed and constructed to study the deposition of super conducting $\mathrm{Nb}$ films. Results obtained with sample $\mathrm{Nb}$ films are promising in that films with "bulk-like" properties were produced. Their RRR and grain size are larger compared to $\mathrm{Nb}$ magnetron sputtered films deposited at the same temperature and also appear less stressed and more randomly oriented. These differences are attributed to the higher energy of the $\mathrm{Nb}$ ions reaching the film, which gives them a high probability of better arranging themselves on the film surface. A filtered UHV arc system was also used to produce quasi microdroplet-free samples. A quantitative estimate of the factor, as obtained with the microdroplet reduction system, is not available at present time. It is due to the fact that the number of the observed microdroplets was below the instrumental detection threshold. It will be necessary to increase sensitivity of the analyzing system.

We acknowledge the support of the European Community-Research Infrastructure Activity under the FP6 "Structuring the European Research Area" program (CARE, contract number RII3-CT2003-506395). 


\section{References}

[1] J. J. Langner: TESLA Report 2000-15. Ed.:D.Proch, DESY 2000.

[2] R.R. Russo et.al.: Proc.X Workshop on RF superconductivity, Tsukuba 2001, KEK Proc. 2003-2, p. 4.

[3] C. Benvenuti et.al.: Physica C 351 (2001) 421.

[4] S. Tazzari et.al.: Proc.XI Worshop on RF Superconductivity, Travemunde 2003, in print.

[5] G. Mesyats: Ectons in a vacuum discharge: breakdown, the spark, and the arc; Nauka, Moscow 2000 (in Russian). 